\title{
Numérique : Nouvelles opportunités, nouvelles inégalités
}

Quelle éducation aux médias numériques pour le rayonnement des cultures locales?

Digital : new opportunities, new inequalities. What digital media education for the spread of local cultures?

Digitales : nuevas oportunidades, nuevas desigualdades. Qué educación digital de los medios de comunicación para la promoción de las culturas locales?

\section{Géraldine Yanon et Francis Barbey}

\section{OpenEdition \\ Journals}

Édition électronique

URL : https://journals.openedition.org/ctd/494

DOI : $10.4000 /$ ctd. 494

ISSN : 2491-1437

Éditeur

Chaire Unesco Pratiques émergentes en technologies et communication pour le développement

Référence électronique

Géraldine Yanon et Francis Barbey, « Numérique : Nouvelles opportunités, nouvelles inégalités », Communication, technologies et développement [En ligne], 6 | 2018, mis en ligne le 18 décembre 2018 consulté le 28 juin 2022. URL : http://journals.openedition.org/ctd/494 ; DOl : https://doi.org/10.4000/ ctd. 494

Ce document a été généré automatiquement le 29 septembre 2020

Communication, technologies et développement 


\section{Numérique : Nouvelles opportunités, nouvelles inégalités}

Quelle éducation aux médias numériques pour le rayonnement des cultures locales?

Digital : new opportunities, new inequalities. What digital media education for the spread of local cultures?

Digitales : nuevas oportunidades, nuevas desigualdades. Qué educación digital de los medios de comunicación para la promoción de las culturas locales?

Géraldine Yanon et Francis Barbey

\section{Et si on commençait par dire que ...}

1 En Côte d'Ivoire, sur une population estimée à environ 24 millions d'habitants, on compte plus de 10 millions d'usagers pour Internet et 21 millions pour le téléphone portable (ARTCI, 2016) ${ }^{1}$. Les usages numériques des communautés locales se font en grande partie autour de ces dispositifs sociotechniques de communication et d'information. Ces pratiques émergentes dans une culture de l'oralité concourent de manière croissante aussi bien à l'amélioration du bien-être, au développement économique, à la consolidation des liens sociaux, à la valorisation des cultures locales qu'à l'engagement citoyen. Ces objets agissent donc sur les sphères sociales, culturelles et politiques (Manuel Castel, 1999). Paradoxalement, leur pénétration en Afrique créée aussi de nouvelles inégalités Nord/Sud, et les disparités entre les villes et les campagnes se font de plus en plus présentes. De même, elle favorise une augmentation de l'offre culturelle étrangère et minimise l'offre locale. La question de la vulgarisation de la culture reste donc un potentiel encore inexploité. Dans un tel contexte, loin de n'être qu'une simple problématique académique, l'éducation aux médias se pose à la fois comme une interrogation sur les TIC et leur façon de nous parler de «nos mondes » et un lieu pour penser l'avenir de nos collectivités. Ceci met en filigrane la question de la culture comme lieu de (sur)vie de toute communauté. 
2 La vulgarisation des TIC oppose deux courants de pensée, optimisme technologique (ethnocentrisme) et pessimisme technologique (dystopie) ${ }^{2}$. Si le premier déterminisme fait de la technologie une baguette magique pour résoudre tous les maux de l'Afrique et " une opportunité pour la diversité des expressions culturelles partout dans le monde » (OIF, 2015), la seconde approche entend ce développement comme une menace pour l'originalité des cultures traditionnelles africaines. Cet article n'objective pas de remettre en cause l'utilité ou non des outils connectés pour les sociétés africaines, mais à rappeler les conditions sous lesquelles ce déploiement technologique peut véritablement contribuer au développement de l'Afrique et à la visibilité de sa culture.

Mobilisant les études sur la sociologie des usages et une recherche documentaire, cet article tente de comprendre les rapports qui se tissent entre les communautés urbaines et villageoises et les objets connectés, ainsi que les inter-actions culturelles qui se créent à partir de leurs différents usages. Ainsi, le modèle techniciste et mercantile de développement du numérique en Afrique ne déplace-t-il pas les inégalités Nord-Sud pour reconduire les logiques impérialistes dans les rapports communicationnels entre les "gens de la ville» et les "gens du village »? Dans quel cadre l'éducation aux médias pourrait-elle intervenir, en considérant les outils numériques comme des outils d'empowerment, et en définissant la communication inter-générationnelle et sociale comme le lieu de préservation et de rayonnement de la culture locale? En partant de la place des outils connectés dans la sociabilité africaine, il s'agit de comprendre comment ces dispositifs impactent l'organisation, les modes de communication des sociétés et établissent une relation particulière aux savoirs. Cet article pose un second regard sur ces dispositifs en tant que moyen de préservation de la culture puisqu'ils participent à la vulgarisation de la diversité culturelle qui s'épanouit aussi bien dans les us et coutumes, les arts, etc. que dans les cadres politiques.

\section{Les outils connectés dans la sociabilité africaine : entre innovations et césures}

4 Au cours de la dernière décennie, la téléphonie mobile s'est rapidement diffusée et implantée sur le continent africain. En Côte d'Ivoire, cette pénétration ne se limite pas aux zones urbaines. En 2016, le pays affiche $113.32 \%$ de taux de pénétration (ARTCI, 2016). On estime le taux de couverture à $100 \%$ dans les zones urbaines contre $31 \%$ dans les villages, ouvrant une porte d'accès à la modernité et à l'information aux zones isolées. L'Internet ne reste pas en marge de ce mouvement même si le taux de couverture reste encore en deçà des $40 \%$. C'est pourquoi de nombreux chercheurs tels que Castells (2000) avec le concept de Network society placent les TIC au cœur des changements qui affectent les sociétés contemporaines.

\section{Des applications innovantes pour une variété et une créativité dans les usages}

L'essor des outils connectés contribue à renforcer les liens sociaux et donne accès à de nouveaux services dont on ne peut nier les aspects positifs. Transfert d'argent via mobile money et e-banking en Côte d'Ivoire, Mpedigree pour traquer les faux médicaments au Ghana, e-commerce du poisson et des denrées périssables au Sénégal et en Afrique du 
Sud, mobile e-learning pour l'alphabétisation, e-gouvernance, accès à l'information, reconnexion des familles dispersées, etc. sont autant de nouvelles formes d'échanges et de communication qui se mettent en place grâce aux outils connectés. Au-delà de l'innovation en termes d'applications et d'usages mobiles, le continent africain intègre dans son développement digital la création de réseaux sociaux numériques. Ces platesformes alternatives locales attirent les Africains et bousculent l'échiquier traditionnel en gagnant de plus en plus de terrain. Si le réseau mondial leader Facebook compte 126.785.000 abonnés en Afrique sur les 2 milliards d'utilisateurs qu'il frôle dans le monde, Ushahidi, Ginger, Mixt, ou encore Eskimi, Yookos et Bandeka $4^{3}$ n'ont rien à envier à ce géant, tant en utilisateurs qu'en initiatives (création de liens, développement de projets éducatifs, commerciaux, etc.). Ces plates-formes «made in Africa " sont en train de devenir de puissants outils de communication disséminés à travers tout le continent. Autour de ces appropriations, l'Afrique invente ses propres « arts de faire » (De Certeau, 1990) et se met en scène. Faisant du web un moyen de diffusion culturel. Ces objets connectés façonnent de nouveaux espaces sociaux à travers lesquels ils mettent les individus au contact d'autres cultures, et induisent de nouvelles pratiques et formes d'inter-activités. Il se crée ici « un monde riche et varié qui élargit les choix possibles, nourrit les capacités et les valeurs humaines, et [est] un ressort fondamental du développement durable des communautés, des peuples et des nations » (Convention sur la protection et la promotion de la diversité des expressions culturelles, 2005).

En Côte d'Ivoire, l'utilisation de plus en plus courante des technologies mobiles laisse entrevoir des usages différentiels entre les «gens de la ville» et les «gens du village ». Dans les campagnes, le téléphone portable tend à maintenir les liens communautaires traditionnels déjà existants : famille, amis, réseaux d'affaires, etc. (Alzouma, 2008). En ville, il est en plus question de s'intégrer dans des cyber-communautés (Ibid). La société numérique permet donc de "s'identifier à un espace élargi, qui dépasse les frontières physiques. Il est aujourd'hui dans un contexte d'hyper mobilité, qui est à la fois matériel, imaginé, et virtuel» (Stebig et Deverin, $2008: 128$ ). Cette pénétration n'est donc pas exempte de retour parfois négatif qui impactent les structures sociales traditionnelles et influencent la culture ainsi que la façon dont les communautés interagissent. Comment ces dispositifs transforment « la morphologie sociale »? (Halbwachs, 1938).

\section{De la « civilisation mondiale multiculturelle » : fondements culturels africains et transformations inhérents au numérique}

7 Si savoir parler aux autres ; savoir écouter les autres ; savoir prendre la parole en public ; savoir danser; savoir chanter; savoir déchiffrer les langages codés, comme ceux des tambours et des objets d'art participent d'une initiation des plus jeunes à la vie sociale et aux règles qui régissent la communauté ainsi qu'une insertion sociale réussie dans les sociétés traditionnelles africaines, la mondialisation de l'offre culturelle rendue possible par le développement des TIC introduit de nouvelles manières de saisir le monde en Afrique. Déjà les pratiques discursives dans cette société de l'oralité se sont raccourcies.

8 Les sujets naguère tabous ou simplement inexprimables aussi bien culturellement que socialement trouvent sur Internet des espaces d'expression, libérant ainsi la parole, qui est généralement l'apanage d'un groupe d'individus (\#éducation sexuelle, \#politique, 
\#viol, \#excision, etc.). La prise de parole formelle en d'autres lieux et circonstances devient, dans ces espaces, plus aisée et surtout n'est pas soumise au poids de la censure.

L'observation de certaines pages ou de chaines YouTube montre combien le Web brise certains tabous en long, en large et en travers pour les rendre à peu près banals. À titre d'exemple la question des menstruations et son hashtag (\#menstruation) en Afrique. Au Congo, Iris Nzolantima, consacre son temps sur les réseaux sociaux Facebook et Twitter (avec $\mathrm{x}$ followers) à briser le silence autour des menstrues. Routines de règles, conseils, récits et anecdotes sont autant de sujets qui alimentent ses pages sur les réseaux sociaux. Outre-Atlantique, l'Américaine Hannah Witton sur sa chaine YouTube, aborde en plus des menstruations la question de la sexualité, du fonctionnement des organes génitaux féminins et le flot de tabous qui leur sont associés. Ou encore en France le blog « Passion menstrues » qui avait été tenu sans complexes par son auteure Jack Parker jusqu'en octobre 2017.

\section{Copie d'écran du site Passion Menstrues}

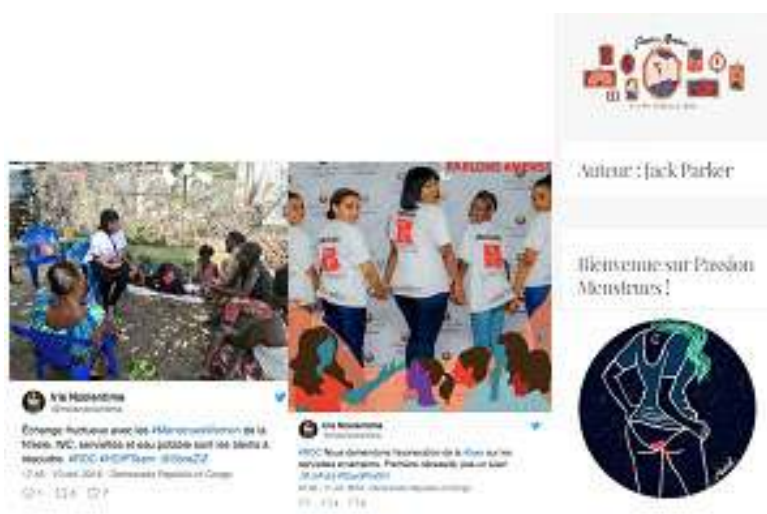

La mondialisation de l'offre informationnelle a introduit une nouvelle donne dans la dynamique médiatique africaine marquée par une présence accentuée des médias étrangers, par conséquent de la culture étrangère. Regarder la télévision, naviguer sur Internet, écouter la radio ou lire les journaux devient de précieux moyens de découvrir d'autres réalités, d'autres modes de vie, codes sociaux, règles politiques, mais aussi d'apprendre ou de se perfectionner. Mais le choc culturel accentué par la fracture culturelle entre le Nord et le Sud laissent une place minime aux contenus culturels africains. Celui-ci «ne représente que $3 \%$ du marché mondial avec 10000 noms de domaines pour toute l'Afrique " (Ballong, octobre 2008). Désormais pour être soi, il faut d'abord être comme les autres, avec leurs identités culturelles. La vie communautaire et individuelle s'en trouve affectée dans la mesure où les TIC apparaissent comme le nœud autour duquel s'organise la société et qu'à des degrés plus ou moins intenses ils affectent ces sociétés. L'originalité des sociétés traditionnelles africaines paraît menacée sous l'influence de la civilisation des médiacultures. Comme le souligne Ki-Zerbo, « le fait que les messages aient été confectionnés dans d'autres contextes culturels en fait des ambassadeurs plénipotentiaires, voire des commandos culturels parachutés dans nos pays » (1990: 58).

11 La volonté de certains jeunes à vouloir ressembler à des personnages qu'ils rencontrent sur Internet, renforce l'idée selon laquelle les outils connectés déstructurent les sociétés et tendent à les uniformiser. Car même s'il ne s'agit pas d'imiter les conduites des acteurs dans la réalité, n'en demeure pas moins qu'il est question « d'essayer des rôles sociaux sur 
un monde imaginaire, afin de s'adapter aux exigences de la socialisation » (Maigret, 2003 : 76). Dans ce même élan de socialisation, les réseaux sociaux sont devenus des journaux intimes et des lieux d'exposition de soi où l'on placarde toutes sortes de choses comme on le ferait avec des posters sur les murs de sa chambre. Ces espaces deviennent des lieux d'expression et de diffusion de sa pensée, de sa culture, de son «soi ». Toute chose qui rassure sur la pérennisation des échanges, mais pas sur l'avenir des cultures africaines. De plus en plus la notion de réseau semble prendre le pas sur celle de communauté dans ce monde globalisé. Pour Castells, le réseau ne serait rien d'autre que la résultante de la perte de la notion de communauté locale induit par les effets du « net » (2000).

Au-delà des questions de l'équilibre informationnel entre le Nord et le Sud, il se (re)joue autour des objets connectés la vie des communautés villageoises comme citadines dans la façon dont les consommateurs culturels fabriquent avec les images qu'ils regardent pendant des heures les objets sociaux. En effet, ces objets apportent des changements radicaux dans les systèmes politiques et communicationnel. À titre illustratif, l'espace numérique comme un bistrot rassemble. À la faveur d'un post (image, son, vidéo, texte) il est possible de débattre d'un aspect de l'actualité sociale directement liée à nos communautés propres. Il est de fait impossible, voire illusoire, sous prétexte de modernité, de ne pas prendre en compte les logiques existentielles et communicationnelles des sociétés africaines, si l'on veut accompagner l'Afrique sur son chemin de «modernité». En d'autres termes, l'Afrique ne peut se développer, en se reniant totalement, pour ne se comprendre que dans la logique des autres (Kiyindou, 2009 : 10-11). L'enjeu est donc de promouvoir une éducation adaptée qui favorisera l'expression des cultures locales africaines.

\section{L'éducation aux médias comme moyen d'interactions porteuses de valeurs communes, de progrès et de promotion de la culture africaine}

\section{De l'expression culturelle africaine ou des réalités africaines}

13 La culture relève des expressions culturelles traditionnelles, le folklore. Ces expressions, élaborées, préservées et transmises de générations en générations participent de la tradition et s'insèrent dans l'identité des peuples et leur patrimoine communautaire. Elles allient savoir, savoir-faire et savoir-être, transmission de valeurs et croyances, et tiennent compte des diverses formes d'émotion dont l'art, la musique, la littérature, etc. Ces savoirs font partie intégrante de l'identité culturelle ou spirituelle d'une communauté et « résultent de l'activité intellectuelle exercée dans un contexte traditionnel [...] et comprennent les connaissances acquises dans les domaines de l'agriculture, de la science, de la technique, de l'écologie et de la médecine ainsi que de la biodiversité »(OMPI). Dans ce sens, la préservation de ces expressions concourt à la promotion de la créativité, à la pérennisation et au renforcement de la diversité culturelle. 


\section{L'éducation aux médias comme moteur de propulsion des expressions africaines}

En partant du principe que l'information produite par les médias est ce qui nous relie au monde, la culture pourrait être ce qui nous relie à notre humanité. Les profondes mutations induites aux objets connectés, mènent à penser avec Corroy, Roche et Savignac que la capacité de l'éducation aux médias à répondre aux défis sociétaux s'impose dans nos républiques africaines, puisque «le monde multimédiatique oblige à réfléchir aux moyens de réduire les fractures sociales et culturelles qui sont susceptibles d'aggraver l'écart entre ceux dotés d'un "capital médiatique et numérique » suffisant et les autres, posant ainsi des enjeux citoyens et plus largement humanistes " (2015). C'est pourquoi dans les sociétés africaines, il faut, par l'éducation aux médias,

«parvenir à une liaison entre les médias modernes et les moyens traditionnels de communication. Ce qui soulève des questions importantes du point de vue de leur influence mutuelle et de leur soutien réciproque. Le principal problème, aussi bien pour les responsables de la politique que pour les praticiens de la communication, est de trouver une formule qui saura préserver les rapports entre les formes de communication traditionnelles et modernes, en conciliant le des problèmes de la communication, $1980: 100$ ).

Dans cette logique, dans une Afrique soumise encore au poids de sa rencontre avec l'extérieur, l'éducation aux médias ne peut pas se limiter aux seules questions d'accès aux TIC, de formation de l'esprit critique et de participation des Africains aux sources de production, mais interroge la capacité des Africains eux-mêmes à puiser les ressources de leur survie dans les lieux où «l'Afrique défie l'avenir » (Ela, 1994: 79) par sa créativité et essaie de se projeter $5^{4}$ dans « sa » modernité. Autrement dit, l'éducation aux médias doit ouvrir de vrais espaces de savoirs, de savoir-faire et de savoir-être, et donc de socialisation et de progrès par la maîtrise des logiques communicationnelles des sociétés traditionnelles africaines. Car, dans une certaine mesure la société de l'information impose une vision du monde en défendant des formes de pouvoirs de domination et des intérêts particuliers (Kiyindou, 2009). Ici, l'éducation aux médias se pense dans une logique où la communauté est la pierre angulaire de tout développement (humain, social, culturel, économique, etc.). Le défi est d'accompagner la créativité des usagers des technologies pour qu'ils intègrent à leurs usages les questions et pratiques liées au développement de la communauté en termes de valeur et de culture au bénéfice de l'Afrique. Ce qui implique un savoir-faire dans l'usage des technologies de la société de l'information (TSI). Cette éducation aux médias aux couleurs africaines valorise la propriété intellectuelle. Dans cette optique, l'Afrique doit être capable non seulement d'écrire ses propres images dans les médias en traitant de sujets endogènes avec des yeux nouveaux et créatifs, mais aussi de renvoyer une image moins caricaturale d'elle au monde.

Le projet qui se dessine à nos yeux parait de toute évidence sociopédagogique et doit être porté progressivement par les gouvernants et les praticiens de cette éducation au profit des populations qui ont accès aux outils de communication. C'est donc un projet qui débute au sein des classes et qui appelle à réfléchir davantage sur les processus de diffusion et de réception - marqués par une prise en compte de la convergence des formats et des transformations ayant cours dans le secteur des technologies - dans un élan de valorisation culturelle. 
17 Par ailleurs, ces dernières années, les technologies numériques permettent une optimisation de la participation démocratique en Afrique. Les exemples sont de plus en plus nombreux. En Côte d'Ivoire par exemple, la présence des acteurs politiques sur les réseaux sociaux ou de groupes dédiés à l'expression replacent les citoyens au cœur du débat politique. Blogs, forums, etc. transforment le Web en un géant agora numérique dans lequel il est désormais possible d'exprimer son opinion. La vulgarisation des outils connectés qui permettent un accès à ces plates-formes démocratiques favorise de fait une participation démocratique de plus en plus grande là où les populations n'ont pas accès aux autorités.

Il faut donc appréhender ces technologies, pour rejoindre la pensée de Garnham, comme des outils à la fois de transformation des relations sociales entre les structures du pouvoir et les gouvernés, mais aussi comme des outils pour penser et se représenter le monde : « tools for thinking and tools for representing the world (Garnham, 2002).

\section{Pour conclure}

«Le numérique est une opportunité pour la diversité des expressions culturelles partout dans le monde » (OIF, 2015)

Le développement des outils connectés en Afrique contribue à l'amélioration des conditions de vie en même temps qu'il pose de nouveaux défis en termes de disparités et de sauvegarde des identités culturelles. S'il est avéré que ces outils sont à la fois des espaces personnels de rendez-vous entre l'homme et la machine, mais aussi entre l'homme et l'homme, ils apparaissent également comme une entrée dans la modernité. Cependant le succès de cette entrée de l'Afrique dans la modernité doit prendre comme cadre de référence ses cultures locales dans leurs différentes expressions, car "aucun pays, aucun peuple au monde ne s'est développé en dehors de ses langues et de ses cultures" (Kiyindou, 2009 : 7). Les investissements en matière de technologies ne doivent pas seulement se limiter à doter les individus d'appareils, mais doivent être suivis d'un accompagnement, d'une éducation.

En Afrique, l'éducation aux médias dépasse le simple cas du mouvement social de publicisation de soi pour rejoindre les TIC dans leur mouvance d'émergence de nouveaux pouvoirs, de globalisation de la culture et de lutte pour la sauvegarde des identités culturelles. C'est pourquoi il convient de promouvoir à travers elle la créativité des jeunes au bénéfice et autour des questions propres à l'Afrique. L'éducation aux médias doit être perçue comme une possibilité de témoigner auprès des jeunes générations « des efforts d'invention et de créativité [...] d'une Afrique dont les médias ne parlent pas » (Ela, 1994 : 79). Il n'est pas encore tard, le grand bradage des cultures africaines n'a pas encore eu lieu et la technologie, grâce à une éducation aux médias adaptée, devient le marché qui peut favoriser le rayonnement de ces identités à l'international. 


\section{BIBLIOGRAPHIE}

Alzouma Gado, « Téléphone mobile, Internet et développement : l'Afrique dans la société de l'information? », Tic \& société, n² 2, vol. 2, 2008. [en ligne] https://journals.openedition.org/ ticetsociete/488, consulté le 30 mai 2017.

Autorité de régulation des télécommunications de Côte d'Ivoire (ARTCI), « Abonnés - Internet », 2017, [en ligne] http://www.artci.ci/index.php/Internet/abonnes-service-internet.html (consulté le 15 mai 2017).

Ela Jean-Marc, Restituer l'histoire aux sociétés africaines. Promouvoir les sciences sociales en Afrique Noire, L'Harmattan, Paris, 1994.

Castells Manuel, La société en réseaux. L'ère de l'information, Fayard, Paris, 2001.

Commission internationale d'étude des problèmes de communication, Voix multiples, un seul monde, La Documentation française /Les Nouvelles Éditions Africaines / Unesco, Paris, 1980.

Fogel Jean-François et Patino Bruno, La condition numérique, Grasset et Fasquelle, Paris, 2013.

Garnham Nicholas, « The information society : myth. Or reality? » Actes du colloque Bugs, globalism and pluralism, Montreal du 24 au 27 avril 2001.

Guibert Gérôme, Rebillard Franck et Rochelandet Fabrice, Médias, culture et numérique. Approches socioéconomiques, Armand Colin, Paris, 2016.

Journal officiel de l'union européenne, « Recommandation du parlement européen et du conseil du 18 décembre 2006 sur les compétences clés pour l'éducation et la formation tout au long de la vie-Annexe : Compétences clés pour l'éducation et la formation tout au long de la vie : un cadre de référence européen ".

Roudier Jérôme (dir.), Médias et cultures en dialogue, Broché, Paris, 2016.

Stebig Jonathan et Devenrin Yveline, «L'appropriation des TIC par les diasporas : Analyse des répercussions potentielles dans les pays d'origine ", Netcom, vol. 22, n 1-2, 2008, pp. 127-144.

Vial Stéphane, «Ce que le numérique change à autrui : Introduction à la fabrique phénoménotechnique de l'altérité », Hermès, nº 68, 2014, pp. 151-157.

Viallon Philippe, « Savoir en ligne versus savoir traditionnel. Peut-on concilier modernité et tradition? ", in Corroy-Labardens Laurence, Barbey Francis et Kiyindou Alain (dir.), Éducation aux médias à l'heure des réseaux sociaux, L'Harmattan, Paris, 2015, pp. 245- 25

\section{NOTES}

1. Parc d'abonnés Internet au 31 décembre 2016 : mobile 10.401.187; fixe : 89.763. Parc d'abonnés téléphonie mobile au 31 décembre $2016: 27.451 .250$. Population ivoirienne estimée en 2016 : 23.865.564

2. Voir Patrice Flichy (2003), L'innovation technique. Récents développement en science sociale. Vers une nouvelle théorie de l'innovation, Paris, Sciences et société, éditions La Découverte. 
3. Ushahidi est un réseau social kenyan qui permet de cartographier en temps réel les vagues de crises sociales et les zones de conflits. Obami est spécialisé dans l'e-learing et permet aux universités et écoles africaines de partager leurs ressources. Yookos, réseau social nigérian à vocation religieuse, Ginger messagerie instantanée. Mixt, Eskimi et Bandeka sont des réseaux sociaux respectivement de l'Afrique du Sud, du Ghana et du Nigéria.

4. La famille, les classes d'âge, les forêts sacrées, es cases d'initiation, l'arbre à palabre. Ces lieux interrogent également la communauté et la rejoint dans l'actualité commune, la nature du rapport intergénérationnel, la place des «Anciens » et du $3^{\mathrm{e}}$ âge, la promotion de l'enfance au sein de la famille et de la société.

\section{RÉSUMÉS}

Le développement du numérique pose à l'Afrique de nouveaux défis en ce qui concerne la préservation et la promotion des expressions culturelles. Profiter des industries créatives implique à chaque citoyen (jeunes, femmes, adultes, minorités, etc.) d'avoir à son actif un large spectre de compétences pour s'adapter durablement à un monde qui ne cesse de se mouvoir par l'inter-connectivité et l'inter- action. Les cultures locales, dans leur diversité et leur unicité, dans leur fonction à la fois sociale et éducatrice participent à ce mouvement. Cet article, en s'intéressant à la place des terminaux mobiles et autres outils connectés dans la sociabilité africaine, interroge la capacité de l'éducation aux médias à amoindrir la fracture culturelle et à soutenir l'expression culturelle africaine comme moteur de développement à travers le numérique, en garantissant la participation citoyenne et démocratique des communautés.

The development of digital poses new challenges for Africa in the preservation and promotion of cultural expressions. Taking advantage of the creative industries means that every citizen (young people, women, adults, minorities, etc.) has a broad spectrum of skills to adapt to a world that is constantly moving through the world. connectivity and interaction. Local cultures, $\mathrm{i}$ their diversity and uniqueness, in their social and educational function, participate in this movement. This article, looking at the place of mobile terminals and other connected tools in African sociability, question the ability of media education to reduce the cultural divide and support African cultural expression as a driver of development across Africa digital, by guaranteeingcitizens anddemocratic participation of communities.

El desarrollo de la tecnología digital plantea a los desafíos de África en cuanto a la preservación y promoción de las expresiones culturales. Disfrutar de las industrias creativas implica a todos los ciudadanos (jóvenes, mujeres, adultos, minorías, etc.) tienen en su haber una amplia gama de habilidades para adaptarse permanentemente a un mundo que continúa moviéndose a través de la inter-conectividad e inter- acción. Las culturas locales, en su diversidad y singularidad, en su función de educador social y ambos participan en este movimiento. En este artículo, centrándose en cambio en los dispositivos móviles y otras herramientas conectadas en la sociabilidad de África, cuestiona la capacidad de la educación de medios para disminuir la brecha cultural y de apoyo a la expresión cultural africana como motor de desarrollo a través de digital, garantizando la participación ciudadana y democrática de las comunidades. Palabras clave: las industrias creativas, la expresión cultural y la diversidad, la alfabetización digital de los medios de comunicación, ciudadanía, comunidad, cultura de masas 
INDEX

Mots-clés : industries créatives, expression et diversité culturelles, éducation aux médias numériques, citoyenneté, communauté locale, médiaculture

Palabras claves : industrias culturales, expresión cultural y diversidad, educación en medios digitales, ciudadanía, comunidad local, cultura de los medios

Keywords : cultural industries, cultural expression and diversity, digital media education, citizenship, local community, media culture

\section{AUTEURS}

\section{GÉRALDINE YANON}

Université catholique de l'Afrique de l'Ouest-Abidjan

FRANCIS BARBEY

Université catholique de l'Afrique de l'Ouest-Lomé 